\title{
A Parametric Device Study for SiC Diodes in Vehicular Applications
}

\author{
Burak Ozpineci $^{1,3}$ \\ Leon M. Tolbert ${ }^{1,2}$ \\ Syed K. Islam ${ }^{1}$ \\ Tim J. Theiss ${ }^{2}$ \\ burak@ieee.org \\ tolbert@utk.edu \\ sislam@utk.edu \\ theisstj@ornl.gov \\ ${ }^{1}$ Department of Electrical and \\ ${ }^{2}$ Oak Ridge National Laboratory \\ P.O. Box 2009 \\ Computer Engineering \\ The University of Tennessee \\ Oak Ridge, TN 37831-6472 \\ ${ }^{3}$ Oak Ridge Institute for Science \\ and Education \\ Oak Ridge, TN 37831-0117
}

Knoxville, TN 37996-2100

\begin{abstract}
Materials and device researchers build switching devices for the circuits researchers to use in their circuits, but they rarely know how and where the devices are going to be used. The circuits people, including power electronics researchers, take the devices as black boxes and use them in their circuits not knowing much about the inside of the devices. The best way to design optimum devices is an interactive design where people designing and building the devices have a close interaction with the people who use them.

This study covers the circuit aspects of the $\mathrm{SiC}$ power device development. As a contribution to the above-mentioned interactive design, in this paper, the device parameters, which need to be improved in order to design better devices, will be discussed.
\end{abstract}

Keywords-Silicon carbide, SiC, device parameters, traction drive, dc-dc power supply, conduction losses, switching losses, PWM.

\section{INTRODUCTION}

Typically, power electronics researchers have to choose off-the-shelf power devices with the specifications best fit for their applications. They, usually, do not have a say about how they would like the device parameters be changed.

Materials and device researchers build switching devices for the power electronics researchers to use in their circuits, but they rarely know how and where the devices are going to be used.

As represented in Fig. 1, a "barrier" exists between the people who design and build power devices and the people who use them in their circuits and systems. Close interaction between the both sides of the barrier is needed to obtain the most performance for devices and systems. With this interaction, the design loop will be closed and the

Prepared by the Oak Ridge National Laboratory, Oak Ridge, Tennessee 37831, managed by UT-Battelle for the U.S. Department of Energy under contract DE-AC05-00OR22725.

The submitted manuscript has been authored by a contractor of the U.S. Government under Contract No. DE-AC05-00OR22725. Accordingly, the U.S. Government retains a non-exclusive, royalty-free license to publish from the contribution, or allow others to do so, for U.S. Government purposes.

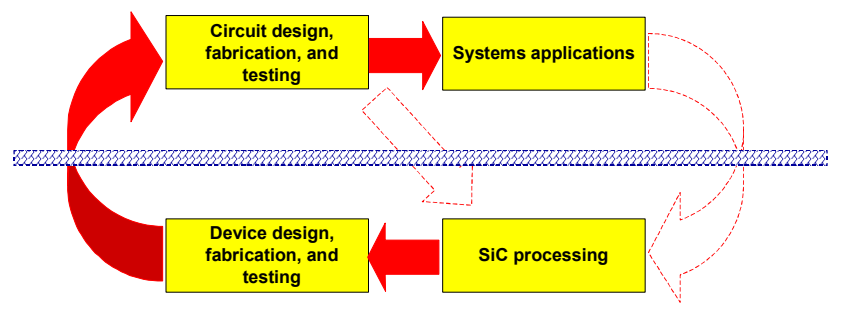

Fig 1. Closing the device design loop.

possibility for building application specific optimum power devices will arise.

Recently, a significant increase in the interest of silicon carbide $(\mathrm{SiC})$ power devices has occurred because of their system level benefits. In the literature, $\mathrm{SiC}$ research is mainly concentrated on the materials and devices aspects $[1$, 2]. Recently, more circuit applications [3, 4] are being published. Moreover, the system level benefits of $\mathrm{SiC}$ are also being evaluated in some recent papers [5-7]. However, $\mathrm{SiC}$ power devices are still in their development stage; therefore, this is a good opportunity at this time to close the loop.

At Oak Ridge National Laboratory (ORNL), a team of materials, device, and power electronics researchers are working together with the University of Tennessee, Auburn University, and Vanderbilt University to build application specific optimum $\mathrm{SiC}$ power MOSFETs. This paper will summarize some of this work.

\section{APPLICATIONS}

This paper is a part of a study where system impact of $\mathrm{SiC}$ power electronics on hybrid electric vehicle (HEV) applications was investigated [5-8]. In the mentioned study, two HEV power converters were identified, modeled, and simulated to show the system level benefits of $\mathrm{SiC}$ power electronics quantitatively. The two selected applications were a dc-dc power supply and a traction drive.

The dc-dc power supply shown in Fig. 2 is an isolated full-bridge dc-dc converter, which is selected mostly because of its high frequency transformer, which provides isolation and additional taps in the secondary to feed more than one converter. 


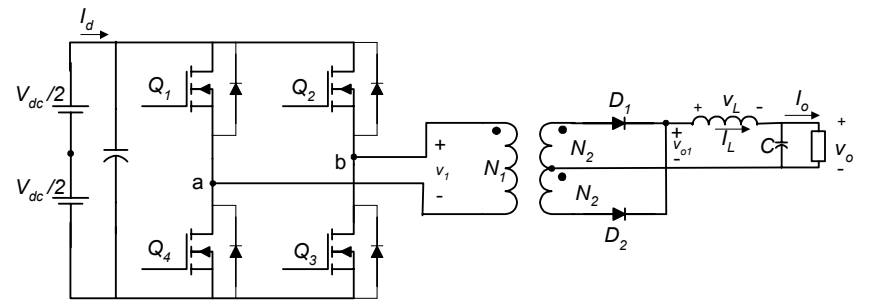

Fig 2. Isolated full-bridge step-down dc-dc converter.

The main traction drive shown in Fig. 3 uses most of the power in an HEV when the vehicle is in motion. A traction drive consists of a battery feeding a three-phase induction machine through a three-phase inverter. Because of the cooling requirements of the power devices in the inverter, usually a large heatsink is required.

In an $\mathrm{HEV}$, any reduction in volume and weight of any component will benefit the efficiency of the vehicle. Because $\mathrm{SiC}$ devices can operate at higher temperatures and they have lower losses, the heatsink volume and weight can be reduced if all $\mathrm{SiC}$ devices are used in all $\mathrm{HEV}$ power converters.

The simulation of these converters using experimental $\mathrm{SiC}$ diode and physics based SiC MOSFET device models showed an increase in the converter efficiency and a decrease in the heatsink mass and volume. The increase in the efficiency of the traction drive was shown to be around $5-10 \%$. Additionally, the reduction in the heatsink can be observed in Tables I and II. Note that for the $20 \mathrm{kHz}$ operation of the dc-dc converter, the $\mathrm{SiC}$ diode needs a bigger heatsink compared to its $\mathrm{Si}$ counterpart. This is because the conduction losses are dominant over the conduction losses at this switching frequency and the conduction losses of the $\mathrm{SiC}$ diode are higher compared with those of the Si diode.. For $100 \mathrm{kHz}$ operation, however, the switching losses are more dominant, then the $\mathrm{SiC}$ diode requires a smaller heatsink compared to its Si counterpart.

Note that for either frequency operation the $\mathrm{SiC}$ inverter requires a smaller heatsink. For the traction drive, all-SiC inverter requires a third of the heatsink required by all-Si inverter.

Improving the related device parameters can increase the efficiency of these converter and the decrease the required heatsink size further.

In the next section these parameters will be identified for SiC Schottky diodes and then necessary suggestions for improvement will be stated.

Note that all these modification suggestions also apply to $\mathrm{Si}$ devices, but the main focus of this study is given to $\mathrm{SiC}$

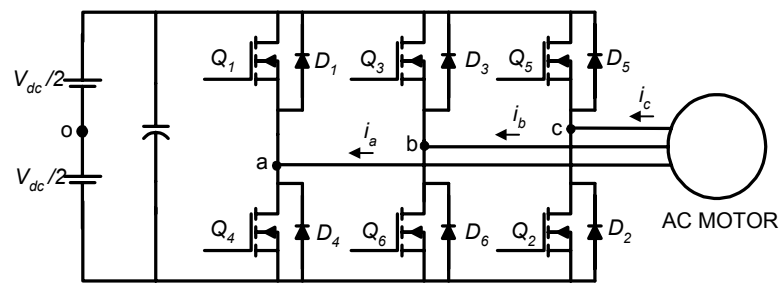

Fig. 3. Three-phase inverter driving an induction machine load.
TABLE I

REQUiREd HEATSINK MASS AND VOLUME FOR THE DC-DC CONVERTER OPERATING AT FULL LOAD

\begin{tabular}{ccccc}
\hline & \multicolumn{2}{c}{$\mathbf{2 0} \mathbf{~ k H z}$} & \multicolumn{2}{c}{$\mathbf{1 0 0 ~} \mathbf{~ H z}$} \\
\cline { 2 - 5 } & Volume $\left(\mathbf{c m}^{\mathbf{3}}\right)$ & Mass $(\mathbf{g})$ & Volume $\left(\mathbf{c m}^{\mathbf{3}}\right)$ & Mass $(\mathbf{g})$ \\
\hline Si diodes & 412 & 1111 & 775 & 2092 \\
SiC diodes & 549 & 1481 & 626 & 1691 \\
Si MOSFETs & 347 & 936 & 1197 & 3232 \\
SiC MOSFETs & 41 & 111 & 205 & 556 \\
Si inverter & 759 & 2047 & 1972 & 5324 \\
SiC inverter & 590 & 1592 & 831 & 2247
\end{tabular}

TABLE II

REQUiRED HEATSINK MASS AND VOLUME FOR THE TRACTION DRIVE OVER THE FEDERAL URBAN DRIVING SCHEDULE

\begin{tabular}{ccc}
\hline & Volume $\left(\mathrm{cm}^{3}\right)$ & Mass $(\mathrm{g})$ \\
\hline Si diodes & 444 & 1200 \\
SiC diodes & 162 & 450 \\
Si MOSFETs & 1554 & 4200 \\
SiC MOSFETs & 444 & 1200 \\
Si inverter & 1998 & 5400 \\
SiC inverter & 606 & 1650
\end{tabular}

power devices.

\section{SiC DIODES}

Some important diode parameters for power electronics systems are the breakdown voltage, on resistance, built-in voltage, peak reverse recovery current, and reverse recovery time.

\section{A. Conduction Loss Parameters}

\section{1) Traction drive}

A diode conduction loss expression for a traction drive inverter shown in Fig. 3 has been derived in [5], and it is repeated below for convenience.

$$
P_{\text {cond }, D 4}=I^{2} \cdot R_{D} \cdot \frac{\S}{\complement}-\frac{1}{3 \pi} M \cos \phi_{\mathfrak{I}}+I \cdot V_{D} \cdot \frac{\S}{\complement 2 \pi}-\frac{1}{8} M \cos \phi_{\mathbf{I}}
$$

where $I$ is the current through the diode,

$M$ is the modulation index for sinusoidal PWM,

$\phi$ is the power factor angle,

$R_{D}$ is the diode series resistance, and

$V_{D}$ is the diode built-in voltage.

This equation consists of two parts, loss associated with the on resistance, $R_{D}$ and loss associated with the built-in voltage drop, $V_{D}$. Diodes with lower $R_{D}$ and $V_{D}$ would be preferable, but these parameters depend on similar device parameters e.g. doping densities. Higher doping density means lower $R_{D}$ but higher $V_{D}$ and lower breakdown voltage, $B V$; therefore, both $R_{D}$ and $V_{D}$ cannot be lowered at the same time, i.e. a trade-off is required.

Consider a $4 \mathrm{H}-\mathrm{SiC}$ Schottky diode with a $B V$ of more than $500 \mathrm{~V}$ for a traction drive.

$$
\begin{gathered}
B V \approx \frac{\mathcal{E}_{r} E_{c}^{2}}{2 q N_{d}}=\frac{1.3511 \times 10^{21}}{N_{d}}>500 \mathrm{~V}, \text { and } N_{d}<2.7 \times 10^{18} \\
\text { where } \quad B V \text { is the breakdown voltage } \\
\mathcal{E}_{r} \text { is the permittivity } \\
E_{c} \text { is the critical electric breakdown field }
\end{gathered}
$$


$q$ is the electron charge

$N_{d}$ is the doping density

The maximum doping density value to sustain the chosen $B V$ is calculated above. The resistance value corresponding to this $N_{d}$ is the minimum $R_{D}$. It cannot be decreased with doping any further; however, the doping density can still be selected lower than this value, which would increase $B V$ and $R_{D}$, and decrease $V_{D}$. Then, the question is: Can modifying $V_{D}$ and $R_{D}$ decrease the conduction losses?

To answer this question, it is required to find how much changes in $R_{D}$ and/or $V_{D}$ will affect the conduction losses.

$I^{2} \cdot R_{D} \cdot \frac{\S}{\complement}-\frac{1}{3 \pi} M \cos \phi_{\mathfrak{I}}^{\cdot}>?<I \cdot V_{D} \cdot \frac{\S}{\complement 2 \pi}-\frac{1}{8} M \cos \phi_{\mathfrak{I}}$

Rearranging terms and assuming $I \neq 0$,

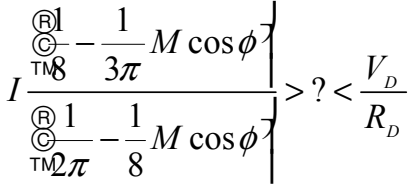

$$
\begin{aligned}
& I \cdot f(M \cos \phi)>?<\frac{V_{D}}{R_{D}} \\
& \text { where } \quad f(M \cos \phi)=\frac{\S \frac{1}{\S}-\frac{1}{3 \pi} M \cos \phi_{\mathfrak{I}}^{.}}{\S \frac{1}{\complement 2 \pi}-\frac{1}{8} M \cos \phi_{\mathfrak{I}}^{\cdot}} \text {, }
\end{aligned}
$$

$M$ is the modulation index, which varies between 0 and $4 / \pi$ (square wave operation), and $\cos \phi$ is the power factor, which varies between 0 and 1 . The power factor of an induction machine is always lagging; for this example calculation, it is assumed to be 0.9 at rated load.

$0 \leq M \leq \frac{4}{\pi}$ and $0 \leq \cos \phi<0.9 \square 0 \leq M \cos \phi<\frac{3.6}{\pi}$

and $f(M \cos \phi)$ varies between 0.787 (no-load) and 0.215 (rated load).

The following example illustrates how to make use of (4). For a particular hybrid electric vehicle traction drive, the rated peak machine current is $136.28 \mathrm{~A}$, which makes $I \cdot f(M \cos \phi)=136.28 \cdot 0.215=29.3 A$. Ignoring the off condition, the minimum device current is the magnetizing current, which is 71A. During the magnetizing current operation, the phase angle is almost $\pi / 2$ radians and the power factor is almost zero, then

\section{$I \cdot f(M \cos \phi)=71 \cdot 0.787=55.9 A$}

Considering (4), the following are some recommendations to maximize the efficiency of a $\mathrm{SiC}$ diode in a traction drive application:

1) If $29.3 \mathrm{~A}>\frac{V_{D}}{R_{D}}$,

then the $R_{D}$ losses are higher at all times, keep the doping density and $R_{D}$ constant because decreasing $R_{D}$ means decreasing $B V$, which would limit the device's application.
2) If $55.9 A<\frac{V_{D}}{R_{D}}$,

then the $V_{D}$ losses are higher at all times, decrease the doping density so that $V_{D}$ will be smaller.

3) If $29.3 \mathrm{~A}<\frac{V_{D}}{R_{D}}<55.9 \mathrm{~A}$,

then the average current of operation will determine the recommended doping density as follows:

a) A drive working close to its rated current value uses the condition, $29.3 A<\frac{V_{D}}{R_{D}}$, where $V_{D}$ losses are higher, decrease the doping density so that $V_{D}$ will be smaller.

b) A drive working at light current loads uses the condition, $\frac{V_{D}}{R_{D}}<55.9 \mathrm{~A}$, where $R_{D}$ losses are higher, keep the doping as it is because decreasing $R_{D}$ means decreasing $B V$, which would decrease the voltage blocking capability of the device.

Fig. 4 displays the above statements on an $R_{D}-V_{D}$ plane.

A commercial SiC Schottky diode $I-V$ characteristics are obtained at different temperatures. From these characteristics, $V_{D}$ and $R_{D}$ values of the diode are calculated. These values are tabulated in Table III and shown as a small rectangular area in Fig. 4. Also shown in Table III is the corresponding $V_{D} / R_{D}$ ratios at different operating temperatures.

At temperatures up to and including $129^{\circ} \mathrm{C}$, the $V_{D} / R_{D}$ ratio is greater than 55.9A, therefore $V_{D}$ losses are higher. At the other temperatures, the ratio is between $29.3 \mathrm{~A}$ and 55.9A. The traction drive will operate close to the rated operation of the induction machine; therefore, consider the comparison with $29.3 \mathrm{~A}$. For all the other temperatures, the ratio is greater than $29.3 \mathrm{~A}$; thus, the $V_{D}$ losses are higher again.

As a conclusion for this case, if the doping concentration, $N_{d}$ for the $\mathrm{SiC}$ diodes in this study is decreased, then $V_{D}$ and the conduction losses decrease. The limit of this decrease is determined by the $V_{D} / R_{D}$ ratio.

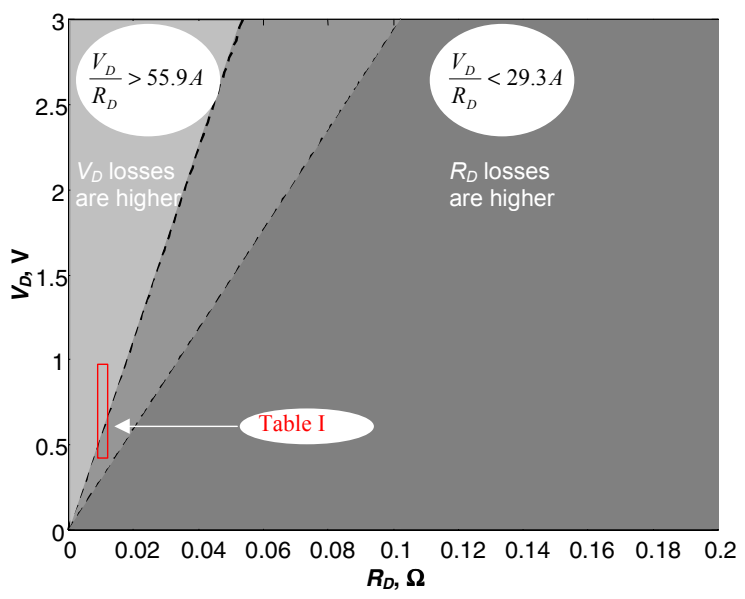

Fig 4 . The $R_{D}-V_{D}$ plane for the traction drive. 
TABLE III

SiC Diode Pwl Model Parameters and $V_{D} / R_{D}$ Ratio

\begin{tabular}{cccc}
\hline $\boldsymbol{T}_{\text {oven }},{ }^{\circ} \mathbf{C}$ & $\boldsymbol{R}_{\boldsymbol{D}}, \mathbf{m} \boldsymbol{\Omega}$ & $\boldsymbol{V}_{\boldsymbol{D}}, \mathbf{V}$ & $\boldsymbol{V}_{\boldsymbol{D}} / \boldsymbol{R}_{\boldsymbol{D}}, \boldsymbol{A}$ \\
\hline 27 & 4.2 & 1.07 & 254 \\
61 & 9.4 & 0.63 & 67 \\
82 & 10.3 & 0.56 & 55 \\
106 & 8.9 & 0.68 & 76 \\
129 & 10.0 & 0.59 & 59 \\
150 & 11.5 & 0.55 & 48 \\
174 & 11.7 & 0.55 & 48 \\
200 & 11.8 & 0.50 & 42 \\
250 & 12.1 & 0.48 & 40 \\
\hline
\end{tabular}

Equation (4) can be used for any sinusoidal PWM application as long as the operation current, power factor, and modulation index information is available.

\section{2). Dc power supply}

The conduction loss expression for the isolated fullbridge dc-dc converter shown in Fig. 2 is as follows:

$$
P_{\text {cond }}=d\left(I_{D} \cdot V_{D}+I_{D}^{2} \cdot R_{D}\right)
$$

where $d$ is the duty ratio of the diode.

Using the same approach as in the previous subsection, the dominant losses can be found as follows:

$$
\begin{gathered}
I_{D}^{2} \cdot R_{D}>?<I_{D} \cdot V_{D} \\
I_{D}>?<\frac{V_{D}}{R_{D}} .
\end{gathered}
$$

The significance of (7) can be summarized as follows:

1) If $I_{D}>\frac{V_{D}}{R_{D}}$,

then the resistive losses are higher, keep the doping and $R_{D}$ constant because decreasing $R_{D}$ means decreasing $B V$, which would decrease the voltage blocking capability of the device.

2) If $I_{D}<\frac{V_{D}}{R_{D}}$,

then the $V_{D}$ losses are higher, decrease the doping so that $V_{D}$ will be smaller.

For different operation condition, the amount of current passing through each device and the voltage across them are calculated. $I_{D}$ varies between $47 \mathrm{~A}$ and $119 \mathrm{~A}$ for a $5 \mathrm{~kW}$ dcdc converter in the HEV simulation, then applying the above criteria,

- If $47 A>\frac{V_{D}}{R_{D}}$, then the first criterion applies.

- If $119 A<\frac{V_{D}}{R_{D}}$, then the second criterion applies.

- If $47 A<\frac{V_{D}}{R_{D}}<119 A$, then it depends on how close the magnitude of the current is to the minimum or maximum values for the majority of the time. For example, if the average load is varying or constant and is in a range between 3.5 and $5 \mathrm{~kW}$, then the current is closer to the upper limit and the second criterion applies. If, on the other hand, the average load is in a range between 2 and $3.5 \mathrm{~kW}$, then the current is closer to the lower limit and the first criterion applies.

This criteria presented here, can be applied to almost any dc-dc converter using $\mathrm{SiC}$ devices.

\section{B. Switching Loss Parameters}

The diode switching losses occur due to the reverse recovery of the diode, which is caused by the stored charge in the depletion region. Schottky diodes are majority carrier devices, so they do not have stored charge. However, they display a characteristic similar to reverse recovery due to the ringing of the parasitics and the internal pn junction caused by the p-rings. The p-rings are used to reduce the large reverse leakage currents.

For Schottky diodes, the switching losses can be reduced either by reducing the parasitic elements or improving the reverse recovery characteristics of the pn junction formed by the p-rings.

A diode switching loss expression has been derived in [5] using Fig. 5:

$$
P_{r r}=f_{c} \frac{V_{R}}{2 S} \frac{\S d I_{F}}{(0)} \cdot \frac{\S S t_{r r}}{1} \cdot{ }^{2}
$$

where $f_{c}$ is the switching frequency,

$V_{R}$ is the reverse blocking voltage,

$I_{F}$ is the forward diode current,

$S$ is the snappiness factor, and

$t_{r r}$ is the reverse recovery time.

In this expression all the parameters except $S$ and $t_{r r}$ are circuit dependent. These two parameters can be expressed [9] in other device parameters for a pn diode as follows,

$$
\begin{aligned}
& S=\frac{2 W_{d}}{b}-1 \\
& t_{r r}=\frac{2 b W_{d}}{D_{n}}
\end{aligned}
$$

where $D_{n}$ is the electron diffusion constant $\left(D_{n}=\frac{k T}{q} \mu_{n}\right)$, $W_{d}$ is the width of the drift region, and $b$ is a distance in the drift region measured from the $p^{+} n^{-}$junction $b=\frac{2 q A D_{n}\left[n(0)-n^{*}\right]}{I_{F}}$ as shown in Fig. 6, $n(0)$ is the carrier density at the $p^{+} n^{-}$junction when the diode is on, and $n^{*}$ is the average carrier concentration in the $n^{-}$region.

Gathering the $S$ and $t_{r r}$ related terms in (8) and inserting (9) and (10), the following is obtained:

$$
\frac{1}{S}{\frac{\S S t_{r r}}{@ S+1} \cdot{ }^{2}}^{2}=\frac{S t_{r r}^{2}}{(S+1)^{2}}=\frac{\left(2 W_{d}-b\right) b^{3}}{D_{n}^{2}}
$$




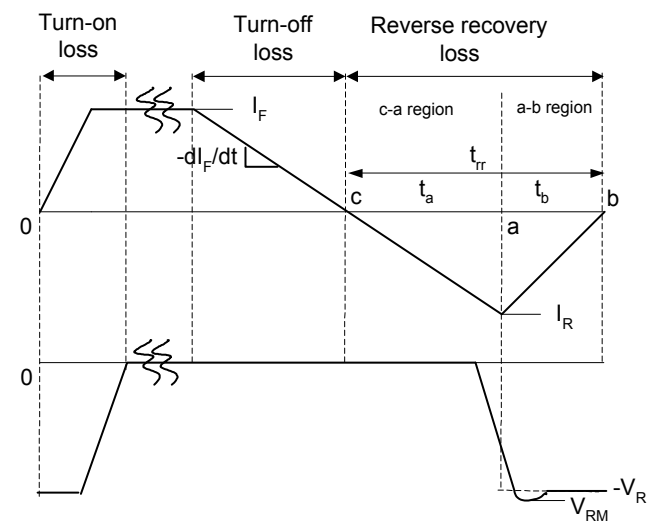

Fig. 5. Typical diode switching waveform

Therefore, decreasing $b$ and/or $W_{d}$ can decrease the switching losses and $b$ can be decreased by decreasing the area and/or $[n(0)-n *]$.

Note that the conclusions here also apply to the $\mathrm{dc}-\mathrm{dc}$ converter, because (8) represents a switching cycle independent of the application.

\section{CONCLUSIONS}

In this paper, losses of the devices in a traction drive are investigated as functions of device parameters. Some modifications to device parameters are suggested to improve the losses in this drive. The next step is for device researchers to consider these suggestions and evaluate the viability of these modifications.

The interaction of device and power electronics researchers will be extremely useful in producing application specific power devices designed for optimum performance. This study is the first step to achieving this goal.

\section{REFERENCES}

[1] M. Bhatnagar and B. J. Baliga, "Comparison of 6H-SiC, 3C-SiC, and Si for power devices," IEEE Trans. on Electron Devices, vol. 40, no. 3, March 1993, pp. 645-655.

[2] K. Shenai, R. S. Scott, and B. J. Baliga, "Optimum semiconductors for high-power electronics," IEEE Transactions on Electron Devices,
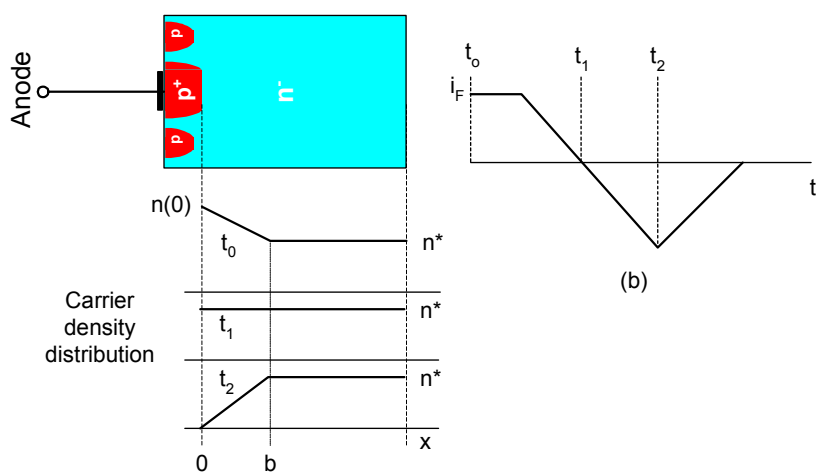

(b)

Fig. 6. Carrier distribution in a diode during turn-off (a) Linearized carrier density distribution of a diode at different time instants

(b) Linearized turn-off current waveform of the diode.

vol. 43, no. 9, Sept. 1989, pp. 1811-1823.

[3] A. Elasser, M. Kheraluwala, M. Ghezzo, R. Steigerwald, N. Krishnamurthy, J. Kretchmer, and T. P. Chow, "A comparative evaluation of new silicon carbide diodes and state-of-the-art silicon diodes for power electronic applications," IEEE IAS Annual Meeting Conference Proceedings, 1999, pp. 341-345.

[4] A. R. Hefner, D. Berning, J. S. Lai, C. Liu, and R. Singh, "Silicon Carbide merged PiN Schottky diode switching characteristics and evaluation for power supply applications," Proceedings of the Annual Meeting of the IEEE Industry Applications Society, 2000, pp. 29482954.

[5] B. Ozpineci, L. M. Tolbert, S. K. Islam, and Md. Hasanuzzaman, "Effects of silicon carbide (SiC) power devices on PWM inverter losses," The Annual Conference of the IEEE Industrial Electronics Society (IECON'01), 2001, pp. 1187-1192.

[6] B. Ozpineci, L. M. Tolbert, S. K. Islam, and F. Z. Peng, "Testing, characterization, and modeling of $\mathrm{SiC}$ diodes for transportation applications, "IEEE Power Electronics Specialists Conference (PESC'02), June 23-27, 2002.

[7] B. Ozpineci, L. M. Tolbert, S. K. Islam, and Md. Hasanuzzaman, "System impact of silicon carbide (SiC) power devices," International Journal of High Speed Electronics and Systems, in press

[8] B. Ozpineci, System impact of silicon carbide power electronics on hybrid electric vehicle applications, A Ph. D. Dissertation, August 2002

[9] B. J. Baliga, Power Semiconductor Devices, PWS Publishing Company, Boston, 1996.

[10] D. A. Grant and J. Gowar, Power MOSFETS-Theory and Applications, John Wiley \& Sons, New York, 1989. 